Presented at the Spring Meeting of the Materials Research Society, San Francisco, California, April 5-9, 1999 and to be published in meeting Proceedings

\title{
Z-Contrast STEM Imaging and EELS of CdSe Nanocrystals: Towards the Analysis of Individual Nanocrystal Surfaces
}

\author{
A. V. Kadavanich, ${ }^{1,2}$ T. Kippeny, ${ }^{2}$ M. Erwin, ${ }^{2}$ S. J. Rosenthal, ${ }^{2}$ \\ and S. J. Pennycook ${ }^{1}$ \\ ${ }^{1}$ Solid State Division, Oak Ridge National Laboratory, P.O. Box 2008, \\ Oak Ridge, Tennessee 37831-6031 \\ 2Department of Chemistry, Vanderbilt University \\ Nashville, Tennessee 37235
}

\begin{abstract}
"The submitted manuscript has been authored by a contractor of the U.S. Government under contract No. DE-ACO5-84OR21400. Accordingly, the U.S. Government retains a nonexclusive, royalty-free license to publish or

reproduce the published form of this

contribution, or allow others to do so, for U.S.

Government purposes."
\end{abstract}

\author{
prepared by \\ SOLID STATE DIVISION \\ OAK RIDGE NATIONAL LABORATORY \\ Managed by \\ LOCKHEED MARTIN ENERGY RESEARCH CORP. \\ under \\ Contract No. DE-AC05-960R22464 \\ with the \\ U.S. DEPARTMENT OF ENERGY \\ Oak Ridge, Tennessee
}

April 1999 


\section{DISCLAIMER}

This report was prepared as an account of work sponsored by an agency of the United States Government. Neither the United States Government nor any agency thereof, nor any of their employees, make any warranty, express or implied, or assumes any legal liability or responsibility for the accuracy, completeness, or usefulness of any information, apparatus, product, or process disclosed, or represents that its use would not infringe privately owned rights. Reference herein to any specific commercial product, process, or service by trade name, trademark, manufacturer, or otherwise does not necessarily constitute or imply its endorsement, recommendation, or favoring by the United States Government or any agency thereof. The views and opinions of authors expressed herein do not necessarily state or reflect those of the United States Government or any agency thereof. 


\section{DISCLAIMER}

Portions of this document may be illegible in electronic image products. Images are produced from the best available original document. 
Z-CONTRAST STEM IMAGING AND EELS OF CDSE NANOCRYSTALS: TOWARDS THE ANALYSIS OF INDIVIDUAL NANOCRYSTAL SURFACES A.V. KADAVANICH ${ }^{*}$, T. KIPPENY*, M. ERWIN*, S. J. ROSENTHAL*, S. J. PENNYCOOK $\dagger$

†Oak Ridge National Laboratory, Solid State Division, Oak Ridge, TN 37831,

*Vanderbilt University, Department of Chemistry, Nashville, TN 37235

ABSTRACT

We have applied Atomic Number Contrast Scanning Transmission Electron Microscopy (Z-Contrast STEM) and STEM/EELS (Electron Energy Loss Spectroscopy) towards the study of colloidal CdSe semiconductor nanocrystals embedded in MEH-PPV polymer films. Unlike the case of conventional phase-contrast High Resolution TEM, Z-Contrast images are direct projections of the atomic structure. Hence they can be interpreted without the need for sophisticated image simulation and the image intensity is a direct measure of the thickness of a nanocrystal. Our thickness measurements are in agreement with the predicted faceted shape of these nanocrystals.

Our unique $1.3 \AA$ resolution STEM has successfully resolved the sublattice structure of these CdSe nanocrystals. In [010] projection (the polar axis in the image plane) we can distinguish $\mathrm{Se}$ atom columns from Cd columns. Consequently we can study the effects of lattice polarity on the nanocrystal morphology. Furthermore, since the STEM technique does not rely on diffraction, it is superbly suited to the study of non-periodic detail, such as the surface structure of the nanocrystals.

EELS measurements on individual nanocrystals indicate a significant amount (equivalent to $0.5-1$ surface monolayers) of oxygen on the nanocrystals, despite processing in an inert atmosphere. Spatially resolved measurements at $7 \AA$ resolution suggest a surface oxide layer. However, the uncertainty in the measurement precludes definitive assignment at this time. The source of the oxygen is under investigation as well.

\section{INTRODUCTION}

CdSe nanocrystals are under investigation for heterojunction optoelectronic devices, such as light-emitting devices (LED's) and photovoltaics (PV's). [1-3] The performance of such devices should be very sensitive to the nature of the junction interface which is the interface between the nanocrystal and the surrounding matrix, usually a conducting polymer. Despite many studies, nanocrystal surfaces are not very well understood in detail since bulk techniques average over all the different surfaces on a nanocrystal and scanning probes do not penetrate the organic ligands on the surface.

In this paper we present the use of a high resolution Scanning Transmission Electron Microscope (STEM) to obtain information about the individual surfaces on nanocrystal. In particular using an annular dark-field (ADF) detector provides Z-Contrast which can resolve the sublattice in CdSe nanocrystals. Hence the polarity of the unit cell can be assigned directly from the Z-Contrast image without resorting to extensive image simulations. Furthermore, since the $\mathrm{Z}$-Contrast image is a direct projection of the atomic positions, the technique is sensitive to aperiodic detail at the surface unlike phase-contrast HRTEM. [4, 5]

Using the sub-nanometer STEM probe for EELS analysis allows chemical analysis on the length scale of a few $\AA$. In principle it should thus be possible to obtain both chemical and structural information from individual nanocrystal surfaces in the STEM. [6]

\section{EXPERIMENT}

Sample Preparation

CdSe nanocrystals were prepared by the method of Murray $[7,8]$ as modified by Peng [9] for size-focussing. The TOPO surface ligands were exchanged with pyridine by heating in anhydrous pyridine for several hours. The nanocrystals were subsequently precipitated with hexanes and dissolved in chloroform. Poly (2-methoxy,5-(2'-ethyl-hexyloxy)-pphenylenevinylene) (MEH-PPV) was prepared by the method of Wudl [10] and dissolved in chloroform. CdSe samples were stored in a glovebox until use, MEH-PPV was stored under argon in brown glass vials. TEM samples were prepared by mixing the MEH-PPV and CdSe solutions and spin-coating onto single-crystal $\mathrm{NaCl}$ substrates (100 surfaces). Typical parameters were $20 \mu \mathrm{l}$ of $2 \mathrm{mg} \cdot \mathrm{ml}^{-1} \mathrm{MEH}-\mathrm{PPV} / 0.05 \mathrm{mg}^{\circ} \mathrm{ml}^{-1} \mathrm{CdSe}$ solution, spun at $2000 \mathrm{rpm}$. The films 
were removed by dipping into a water surface, whereupon the film floats onto the surface as the $\mathrm{NaCl}$ dissolves away. The floating films were picked up with lacey carbon coated copper TEM grids (Ted Pella Co.). Film thicknesses were typically in the range from 150-200 $\AA$ as judged from the optical absorption of identical films spun onto glass slides. Attempts to directly measure the film thickness using AFM have so far not yielded reliable results but indicate significant roughness on the order of $50 \AA$ consistent with ellipsometry measurements. Specimens for EELS analysis were prepared in a glovebag (Aldrich Atmosbag) purged with dry nitrogen and stored under nitrogen or argon. Specimens were loaded with a glovebag attached to the microscope under nitrogen flow directly from a standard gas cylinder. Specimens for Z-contrast imaging were prepared in air, stored under argon, and loaded in air.

STEM

EELS analysis was performed in a Vacuum Generators (VG) model HB501 STEM operating at $100 \mathrm{kV}$ with an ultimate resolution of $2.2 \AA$. However, to optimize EELS data collection, the probe was run with low excitation of the condenser lens and the exact probe size was not measured. A parallel EELS system using a CCD detector with near single-electron sensitivity was employed. The system is described in more detail in [6]. Data were analyzed using Wavemetrics Igor Pro 3.13. Pre-edge backgrounds were fitted to a power law function and subtracted. Integration of stripped edges used Igor Pro's algorithm based on the trapezoidal method.

For scanning individual nanocrystals, the EELS signal was collected with the STEM in area imaging mode at a magnification such that only one nanocrystal was in the field of view. In this case it was possible to compensate for specimen drift by observing the image and adjusting the electronic image shifts so as to keep the nanocrystal centred on the screen. An area immediately adjacent to each nanocrystal was scanned at the same magnification for the same amount of time to obtain the MEH-PPV blank signal. Time series were acquired to gauge sample damage due to the beam. The oxygen $\mathrm{K}$-edge signal at $532 \mathrm{eV}$ was found to decay over time, but the rate of decay was similar for both nanocrystals and MEH-PPV.

For.linescans it is not possible to observe the image while scanning. In this case an image was taken before and after scanning and scans with excessive image drift (more than $-10 \AA$ ) were rejected. The actual linescan is obtained by scanning the probe at a constant rate across the specimen in the $x$-direction only. EELS spectra are acquired successively at fixed signal integration times resulting in a series of EELS measurements at different points along the line.

Z-Contrast imaging was performed in a VG HB603 STEM operating at 300kV with a nominal resolution of $1.3 \AA$. Raw images were deconvoluted using a Maximum Entropy $[11-13]$ algorithm running on a dedicated PC with a custom coprocessor card. The details are described elsewhere. [4] For presentation, image brightness/contrast was adjusted in NIH Image 1.61.

For a single atom, the image contrast is proportional to the square of the atomic number $\left(Z^{2}\right)$. For a crystalline specimen in a channeling orientation, the intensity also depends on the thickness of an atomic column along the beam direction. The dependence is non-linear but monotonic and can be calculated from the known crystal structure for any given channeling direction. For typical nanocrystal sizes $(<80 \AA)$ the dependence is nearly linear so that intensities can be used for comparing relative thicknesses between atomic columns. Intensities are measured in NIH Image by summing the values of all pixels in a chosen region and subtracting out the average value of the background intensity.

\section{RESULTS/DISCUSSION}

EELS

The expected nitrogen $\mathrm{K}$-edge at $400 \mathrm{eV}$ arising from pyridine was not observed. This is consistent with other experiments suggesting that the pyridine is removed during the filmforming process. [1, 3]

The EELS spectrum in the vicinity of the oxygen K-edge for a single nanocrystal with a diameter of $65 \AA$ on the long axis is shown in the top of Figure 1. The nanocrystal lattice is not resolved under the imaging conditions used for the EELS measurement, so the size measurement is not very precise. The short axis diameter is approximately $60 \AA$.

The corresponding spectrum for MEH-PPV is shown in the bottom of the figure. The stripped oxygen edges immediately indicate that the nanocrystal scan contains more oxygen, despite the fact that the amount of MEH-PPV is less due to the volume excluded by the 
nanocrystal.

To convert the integrated intensity of the MEH-PPV oxygen edge into amount of oxygen we take the volume of MEH-PPV in the scan (area - thickness) and multiply it by the density of MEH-PPV. The latter value is not well known. For our calculations we approximate it as 1 $\mathrm{g}^{\bullet} \mathrm{cm}^{-3}$. Undissolved large pieces of MEH-PPV are buoyant in chloroform (density $=1.5{\mathrm{~g} \cdot \mathrm{cm}^{-3}}^{-3}$ so we believe our estimate to be valid to within a factor of two. However, this is our dominant source of error in the quantitation. This yields a conversion of edge intensity to number of oxygen atoms for a given scan.

To calculate the amount of oxygen on the nanocrystal surface ${ }^{1}$ we first subtract out the contribution of the MEH-PPV matrix. To account for the volume excluded by the nanocrystal we approximate it as an ellipsoidal spheroid. We also use the spheroidal approximation to calculate the nanocrystal surface area. Using the density of surface sites of wurtzite $\mathrm{CdSe}(100)$ and (001) surfaces (the predominant surfaces in CdSe nanocrystals, both with about 0.064 sites $\AA^{-2}$ ) we calculate the total number of surface sites (both $\mathrm{Cd}$ and $\mathrm{Se}$ ) on the nanocrystal. The coverage is defined as the ratio of oxygen atoms to available surface sites, 1 monolayer (ML) indicating all sites oxidized. This somewhat overestimates the coverage as edge atoms should have two surface dangling bonds capable of bonding to oxygen. However, given the other uncertainties we do not expect this to contribute significantly to our measurement error. For the nanocrystal shown in Figure 1, we obtain 1.3ML of oxygen on the nanocrystal surface. Another measurement in the same specimen gave a coverage of $0.5 \mathrm{ML}$.

At present these are our only quantifiable measurements. Given the many uncertainties in the quantitation and the poor statistics, the safest conclusion is that a large amount of oxygen occurs on the nanocrystal surface and the coverage is on the order of $1 \mathrm{ML}$. This is consistent with previous EELS measurements on ensembles of nanocrystals. The source of the oxygen is unknown at this time. Likely candidates are TOPO from the original synthesis, the MEH-PPV matrix, or the water bath used in the specimen preparation. We are currently pursuing control experiments to test these hypotheses.

The measurements shown so far still average over all the surfaces of a nanocrystal. To

${ }^{1}$ Since CdSe nanocrystals show good crystallinity in HRTEM, Z-STEM and diffraction measurements we assume the majority of the oxygen associated with nanocrystals to occur at the nanocrystal surface 
test the feasibility of analyzing individual nanocrystal surfaces we have performed linescans across individual nanocrystals.

One such linescan is shown in Figure 2, with 17 scan increments in the line, acquiring for $4 \mathrm{~s}$ in each step. Performing the pre-edge background fitting and subtraction in a similar fashion but without converting to absolute numbers of oxygen atoms, we obtain the position dependent oxygen concentration profile shown in the bottom part of Fig. 2. The total drift in the image for the entire linescan was about $10 \AA$, predominantly to the right, so that the positional uncertainty in each step is less than $1 \AA$.

The depletion of oxygen at the center of the nanocrystal is due to the exclusion of MEH-PPV. The slight increase at the edges of the nanocrystal sugestive of a thin oxide shell viewed in projection, but within the measurement uncertainty it is not significant. Hence no firm conclusion can be drawn although it does demonstrate the potential for surface specific chemical analysis. It should be noted that the acquisition parameters correspond to detecting less than 10 atoms of oxygen within a scan increment on the MEH-PPV. We expect to improve the measurement uncertainty by further fine-tuning the experimental parameters. In particular by reducing the specimen drift it will be possible to integrate longer in each individual scan and improve the signal-to-noise ratio. Furthermore acquiring a longer range of the pre-edge background should improve the extrapolation into the edge region, resulting in more reliable integration.

\section{Z-STEM}

The Z-STEM image of a nanocrystal near [010] zone axis orientation is shown in Figure 3. Panel (a) shows the raw image and panel (b) is the Maximum Entropy Reconstruction of the object function with the point

Fig. 3:

a) Z-STEM image of a CdSe nanocrystal near [010] zone axis orientation.

b) Maximum Entropy Reconstruction of the object function

c) enlarged view of the area delimited by the box in (b). The dumbbell pairs can be resolved into the atomic columns $1.5 \AA$ apart.

d) line scan in the direction of the arrow in (c) showing the intensity in the two columns. The intensity distribution closely matches the expected contrast for $\mathrm{Cd}$ relative to $\mathrm{Se}(2: 1)$. Hence the directions of the $<001>$ and $<00-1>$ lattice vectors can be assigned to the image as shown.
Fig. 2:

Top: DF image of a single nanocrystal with the linescan indicated by the horizontal line. The marker in the center indicates the length of each scan step and thus the area integrated for each EELS measurement.

Bottom:

The oxygen concentration profile calculated from the EELS oxygen K-edge intensity at each scan step. The depletion at the center is attributed to the excluded volume of MEH-PPV.

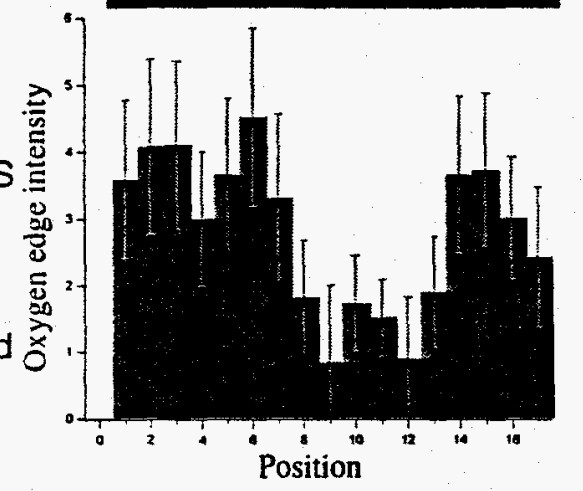


spread function of the microscope removed. Panel (c)

shows a magnified view of the area in (b) indicated by the square. The dumbbell pairs of $\mathrm{Cd}$ and Se columns spaced $1.5 \AA$ apart are just resolved. The different intensities indicate that the Cd comprises the top right column of the dumbbell pair. This is more evident from the integrated intensity profile in (d) of the area indicated. Not all columns are as well resolved within this image. This could indicate strain in the lattice, but it could also be due to image noise. Based on the contrast in the atomic column the $<001>$ lattice vector direction is assigned as up in the image.

It is assumed that the $\mathrm{Cd}$ and Se columns are of equal thickness within each dumbbell column. One may reasonably expect them to differ by one atom at the entrance and exit surfaces each, depending on the exact nature of the surface termination. For the nanocrystal shown the thickness should be on the order of $15-18$ atoms so the maximum error would be $13 \%$ which cannot account for the contrast difference observed. Hence the assignment of the elemental identities is clear-cut.

The overall noise level in the image is still too large to unambiguously determine atomic positions in the surface region. There is also some overlap with a second nanocrystal in the top
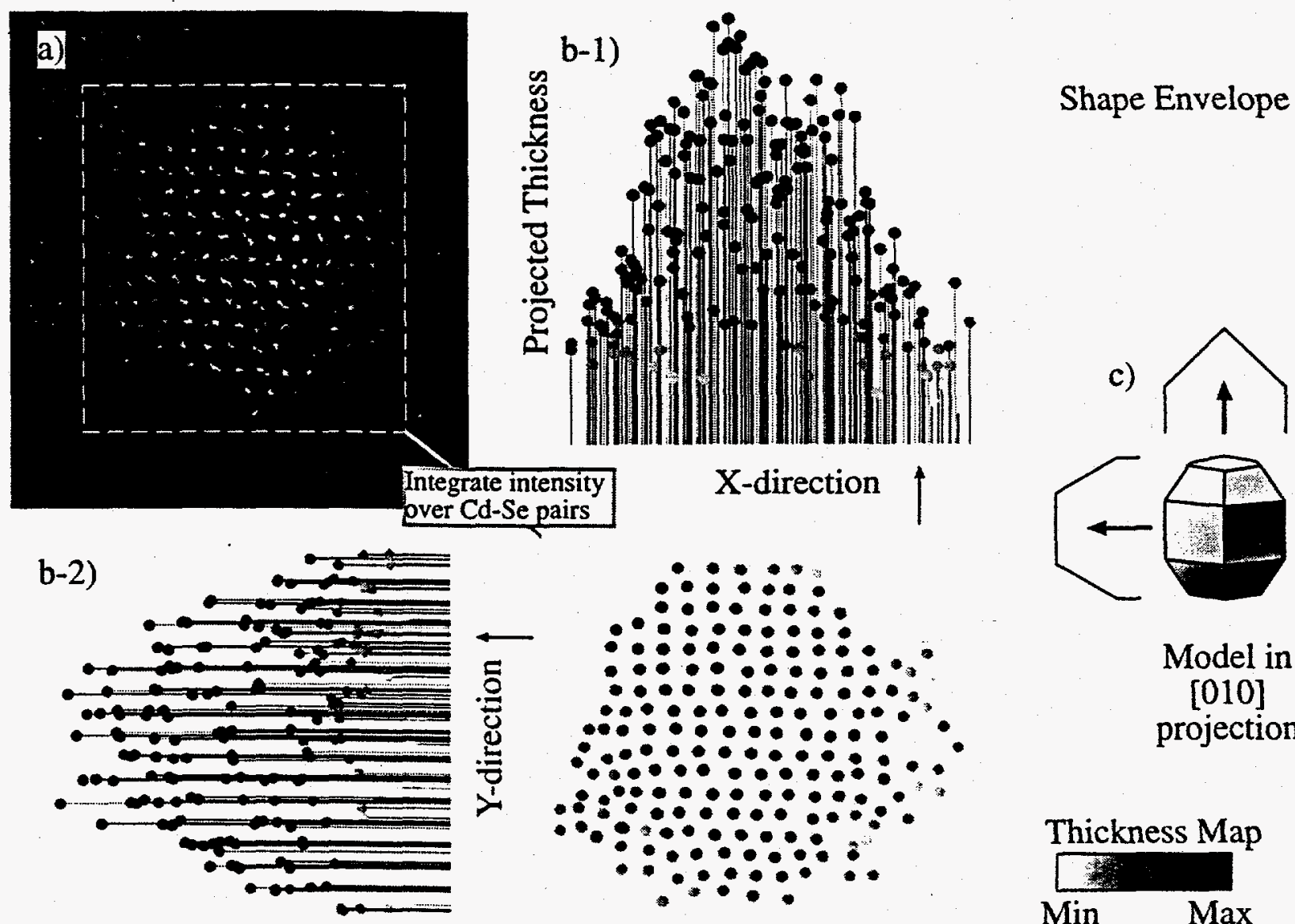

Projected Thickness

Fig. 4: 3-D Analysis of CdSe nanocrystal

a) the Z-STEM image after Maximum Entropy Reconstruction

b) Thickness map (raw intensity) on a grayscale after integrating the intensity in each dumbbell pair (or otherwise resolvable feature in the image). The indicated thickness is not corrected for the thickness dependence of the contrast, so values are not absolute.

b-1) Projection of the thickness map along the horizontal direction in the image. The envelope of the thicknesses should correspond to the shape envelope of the nanocrystal.

b-2) Same as (b-1) in the vertical direction.

c) Schematic of the expected shape in [010] projection based on previous HRTEM studies and the expected shape envelopes in each projection. 
left corner of the image. The right edge of the nanocrystal seems to exhibit some disorder, but such an assignment would be tentative at this time.

If we integrate the total intensity of each dumbbell pair we obtain a spatially resolved thickness map, since the intensities are then independent of composition. This analysis is shown in Figure 4. The thickness envelope corresponds to the expected shape based on previous HRTEM studies on such nanocrystals. [14] However, in this case the three-dimensional information is obtained in concert with the 2-dimensional projection, directly from the image.

\section{CONCLUSIONS}

Using sub-nanometer STEM probes it is possible to obtain chemical information on individual nanocrystals from spatially resolved EELS measurements. Applying this to CdSe nanocrystals embedded in MEH-PPV reveals oxygen equivalent to surface coverage on the order of 1 monolayer. Linescans suggest a surface oxide layer but the assignment is ambiguous at present.

Z-Contrast STEM is capable of resolving the lattice polarity in CdSe nanocrystals and can also provide thickness information directly from the image. Surface detail should be resolvable but the currently available data is not of sufficient quality yet.

\section{ACKNOWLEDGEMENTS}

This research was sponsored by the Division of Materials Sciences, U. S. Department of Energy under contract No. DE-AC05-96OR22464 with Lockheed Martin Energy Research Corp.

AVK gratefully acknowledges the assistance of P.D. Nellist, B.E. Rafferty, M. F. Chisholm, Y. Yan and G. Duscher in the operation of the STEM and EELS systems. We wish to thank C. Chen and G. Jellison for ellipsometry and J. Taylor for AFM measurements on films.

\section{REFERENCES}

Additional Figures are available at http://home.earthlink.net/topchem/MRS_Spring99 until at least Dec. 1999.

1. Schlamp, M.C., Peng, X., and Alivisatos, A.P., Improved efficiencies in light emitting diodes made with $\mathrm{CdSe}(\mathrm{CdS})$ core/shell type nanocrystals and a semiconducting polymer. Journal of Applied Physics, 1997.82 (11): p. 5837-42.

2. Colvin, V.L., Schlamp, M.C., and Alivisatos, A.P., Light-Emitting Diodes Made From Cadmium Selenide Nanocrystals and a Semiconducting Polymer. Nature, 1994.370 (6488): p. 354-357.

3. Greenham, N.C., Xiaogang, P., and Alivisatos, A.P., Charge separation and transport in conjugatedpolymer/semiconductor-nanocrystal composites studied by photoluminescence quenching and photoconductivity. Physical Review B (Condensed Matter), 1996.54 (24): p. 17628-37.

4. Pennycook, S.J., Jesson, D.E., McGibbon, A.J., and Nellist, P.D., High angle dark field STEM for advanced materials. Journal of Electron Microscopy, 1996.45 (1): p. 36-43.

5. Nellist, P.D. and Pennycook, S.J., Accurate structure determination from image reconstruction in ADF STEM. Journal of Microscopy-Oxford, 1998 : p. 159-170.

6. Duscher, G., Browning, N.D., and Pennycook, S.J., Atomic column resolved electron energy-loss spectroscopy. Physica Status Solidi A, 1998. 166 (1): p. 327-42.

7. Murray, C.B., Norris, D.J., and Bawendi, M.G., Synthesis and Characterization Of Nearly Monodisperse CdE $(E=S, S e, T e)$ Semiconductor Nanocrystallites. Journal Of the American Chemical Society, 1993. 115 (19): p. 8706-8715.

8. Bowen Katari, J.E., Colvin, V.L., and Alivisatos, A.P., X-ray Photoelectron Spectroscopy of CdSe Nanocrystals with Applications to Studies of the Nanocrystal Surface. Journal of Physical Chemistry, 1994. 98 (15): p. 4109-17.

9. Peng, X.G., Wickham, J., and Alivisatos, A.P., Kinetics of II-VI and III-V colloidal semiconductor nanocrystal growth: "Focusing" of size distributions. Journal Of the American Chemical Society, 1998. 120 (21): p. 5343-5344.

10. Wudl, F. and Srdanov, G., Conducting Polymer formed of Poly(2-Methoxy, 5-(2'-Ethyl-Hexyloxy)-pPhenylenevinylene), in United States Patent. 1993: United States of America.

11. Burch, S.F., Gull, S.F., and Skilling, J., Image restoration by a powerful maximum entropy method. Computer Vision, Graphics, and Image Processing, 1983. 23 (2): p. 113-28.

12. Skilling, J. and Bryan, R.K., Maximum entropy image reconstruction: general algorithm. Monthly Notices of 
the Royal Astronomical Society, 1984. 211 (1): p. 111-24.

13. Skilling, J. and Sibisi, S. Fundamentals of MAXENT in data analysis. in Invited and Contributed Papers from the Conference. 1990. Chilton, UK: IOP.

14. Shiang, J.J., Kadavanich, A.V., Grubbs, R.K., and Alivisatos, A.P., Symmetry of Annealed Wurtzite CdSe Nanocrystals: Assignment to the $C_{3 v}$ Point Group. Journal of Physical Chemistry, 1995. 99 (48): p. 1741717422. 